\title{
Metastatic breast carcinoma to the pituitary gland that presented as diabetes insipidus: a case report
}

\begin{abstract}
Background: Bones, lungs and liver are the most common sites of primary breast tumors. Metastases to the pituitary gland are rare and usually indicate widespread malignant disease. The more frequent manifestations of pituitary gland involvement are diabetes insipidus and visual disturbances.

Case report: This is a 45-year-old woman who was presented with headache, visual disorders, polyuria, polydipsia, significant hypernatremia and dyspnea. The clinical examination revealed a palpable mass in the right breast, while the imaging evaluation showed extensive lung, liver, brain and bone metastases and a metastatic lesion in the pituitary gland, which was compatible with the symptoms of the patient. She received desmopressin for diabetes insipidus control, and underwent whole brain - orbital irradiation and chemotherapy, with resolution of her symptoms and partial remission of the disease. This case shows that diabetes insipidus may be the first manifestation of metastatic breast cancer and early detection and treatment, can improve the associated symptoms.
\end{abstract}

Keywords: breast cancer, pituitary gland, diabetes insipidus
Volume 8 Issue 2 - 2017

loannis Samaras,' Konstantinos Tsapakidis,' Eleni Maragouli,' Eleni Sogka,' loannis Litos,' Maria Tolia, ${ }^{2}$ Vasileios N Papadopoulos,' Konstantina Papacharalampous, ${ }^{3}$ George Kyrgias, ${ }^{2}$ GK Koukoulis, ${ }^{3}$ Georgios Papatsimpas'

'Department of Medical Oncology, University of Thessaly, University Hospital of Larissa, School of Medicine, Greece ${ }^{2}$ Department of Radiotherapy, University of Thessaly, School of Health Sciences, Faculty of Medicine, Greece ${ }^{3}$ Department of Pathology, University of Thessaly, School of Health Sciences, Faculty of Medicine, Greece

Correspondence: loannis Samaras MD, Department of Medical Oncology, University of Thessaly, University Hospital of Larissa, School of Medicine, Biopolis, Larissa 4 I I 10, Greece, Tel 3.02E+I I, Fax 3.02E+ I I, Email jnsamaras@gmail.com
Abbreviations: DDAVP, desmopressin acetate(DDAVP); $\mathrm{ADH}$, antidiuretic hormone (ADH); HER-2, human epidermal growth factor receptor 2 (HER-2); CT, computed tomography (CT); ACTH, adrenocorticotropic hormone

\section{Introduction}

The most common metastatic sites of primary breast tumors are bones, lungs and liver. ${ }^{1-4}$ Metastases to the pituitary gland are rare. ${ }^{1,2}$ They are asymptomatic in $7 \%$ of cases, ${ }^{1,5}$ but when signs and symptoms appear, these are mainly diabetes insipidus (when the pituitary posterior lobe is involved), headache, visual disturbances, ${ }^{6,7}$ and endocrine abnormalities.

Metastases to the pituitary gland as the first manifestation of the disease are uncommon ${ }^{8,9}$ and maybe the only site of metastases. ${ }^{10,11}$. Sometimes, it is difficult to distinguish between malignant and benign lesions in the pituitary gland. When the pituitary metastases occur, it is important to control the local disease, particularly to alleviate the symptoms. ${ }^{3,12,13}$ The prognosis depends on the location and extent of the primary tumor, rather than the pituitary lesion. ${ }^{1}$ As survival of metastatic breast cancer improves, pituitary metastases may appear more frequently. $3,14,15$

\section{Case presentation}

A 45-year-old woman was admitted to the Emergency Department of our hospital on May 2015 due to headache (retrobulbar), visual disorders (blurred vision), polyuria (14lt/day), polydipsia, dyspnea, weakness, weight loss, progressing during the last months. The patient, originated from Albania, had a free personal medical history and in her family history there is an aunt with breast cancer, diagnosed at the age of 60. Physical examination revealed a palpable mass in the right breast, and palpable liver. The laboratory tests showed normochromic normocytic anemia $(\mathrm{Hb}=7.4 \mathrm{~g} / \mathrm{dl}, \mathrm{Hct}=23.4 \%)$, elevated cholestatic enzymes $(\gamma \mathrm{GT}=423 \mathrm{U} / \mathrm{l}, \mathrm{ALP}=237 \mathrm{IU} / \mathrm{l})$ and significant hypernatremia (serum sodium $=160 \mathrm{mmol} / \mathrm{l}$ ). The patient initially underwent a mammography and then a breast MRI, which showed a $38 \times 21 \mathrm{~mm}$ lesion in the lower inner quadrant of the right breast. Computed abdomen and chest tomographies were performed and revealed several pulmonary and liver metastases. Bone scan showed multiple secondary lesions. Hypernatremia, polyuria and polydipsia raised suspicion of diabetes insipidus, confirmed by a low urine specific gravity $(<1005)$. A fluid deprivation test was then performed to determine the cause of polyuria (the specific gravity and osmolality of urine weren't increased, so we didn't have a primary polydipsia) and we continued administering of desmopressin (DDAVP) in which our patient responded by reducing the polyuria $>50 \%$, so it was central diabetes insipidus (defect in ADH production) and not a nephrogenic one (defect in the kidneys' response to ADH). Core needle biopsy from the breast lesion concluded in a diagnosis of a grade III ductal adenocarcinoma (Figure 1), estrogen and progesterone receptorpositive with overexpression of the HER-2 protein. Ophthalmological assessment revealed bilateral exudative retinal detachments, due to bilateral retinal metastases.

The patient then underwent a brain $\mathrm{CT}$ scan, with a $2 \mathrm{~cm}$ homogeneously enriched focal lesion emerging in the right frontal lobe, compatible to metastasis, and several smaller scattered in the brain parenchyma. Parallelly, an $8 \mathrm{~mm}$ enriched lesion in the pituitary fossa was also depicted, compatible either with a pituitary adenoma or a metastasis. To clarify the nature of the lesion, pituitary MRI was performed, which showed an $8.5 \times 6.5 \mathrm{~mm}$ mass lesion in the pituitary stalk, which argued for metastatic disease, with hemodynamic pattern similar to the metastasis in the right frontal lobe (Figure 2). The 
MRI findings confirmed the diagnostic suspicion of central diabetes insipidus, as was shown by the signs and symptoms of the patient (hypernatremia, polyuria, polydipsia, low urine specific gravity), which is a result of hyposecretion of antidiuretic hormone. An increase in prolactin levels, $1115 \mathrm{mIU} / \mathrm{L}$ (normal levels are less than $500 \mathrm{mIU} / \mathrm{L}$ ) was also found. Thyroid function and ACTH levels were normal.

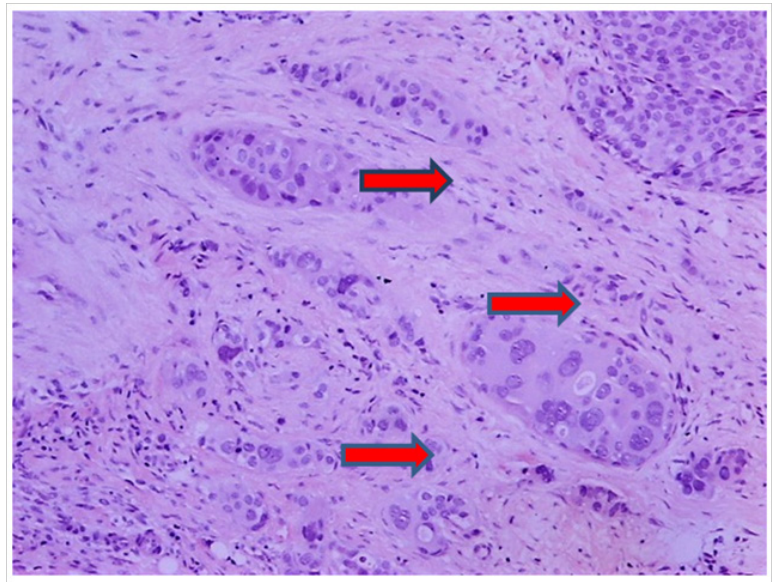

Figure I Ductal carcinoma, grade III, (NOS) not otherwise specified (NOS) or (NST) no special type. Irregular infiltration of stroma by sheets, cords and individual neoplastic cells. Big, pleomorphic cells with big, hyperchromatic or vesicular nuclei, with prominent nucleoli. Tubular formation are absent.

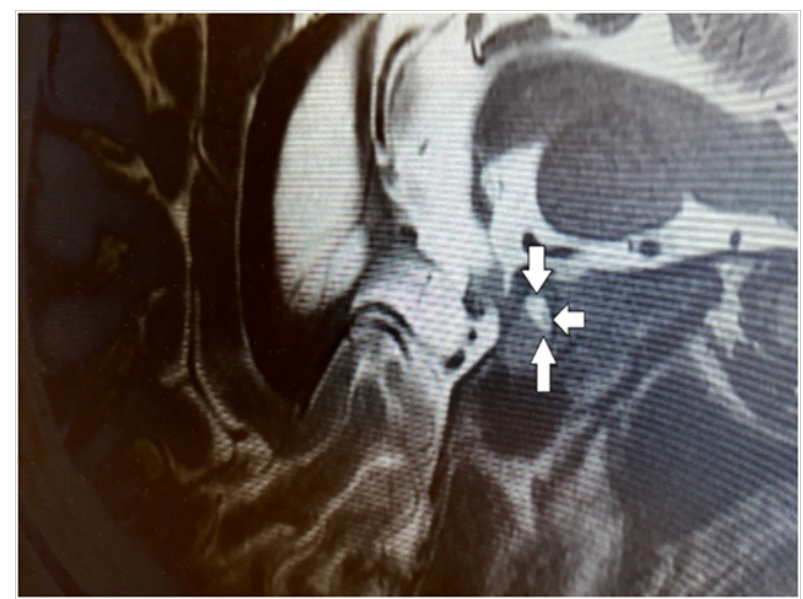

Figure 2 Sagittal pre-contrast TI-weighted MR image showing normal pituitary gland with tumor located in the in the pituitary stalk pointed by arrows.

The written informed consent of the patient was obtained and she underwent whole brain and orbital irradiation (3000 cGy in 10 fractions, daily, from Monday to Friday) (Figure 3). At the same time, the patient received desmopressin, with a gradually increasing dosage (initially $60 \mathrm{mcg}$ daily and then $120 \mathrm{mcg}$ daily), which resulted in controlling symptoms of central diabetes insipidus. Consequently, the patient was treated with immunotherapy and chemotherapy (pertuzumab, trastuzumab, docetaxel for 8 cycles every 3 weeks and then continued on with pertuzumab and trastuzumab). Anti-HER's-2 antibodies, plus the receptor activator of nuclear factor $\kappa \mathrm{B}$ ligand (RANKL) inhibitor (denosumab) is still administering. The patient is still alive, with full control of central diabetes insipidus and with partial remission of the pituitary tumor. Diabetes insipidus may occur in the course of the disease in a patient with metastatic breast cancer. The particularity of our patient, is that the disease appeared with symptoms of diabetes insipidus.

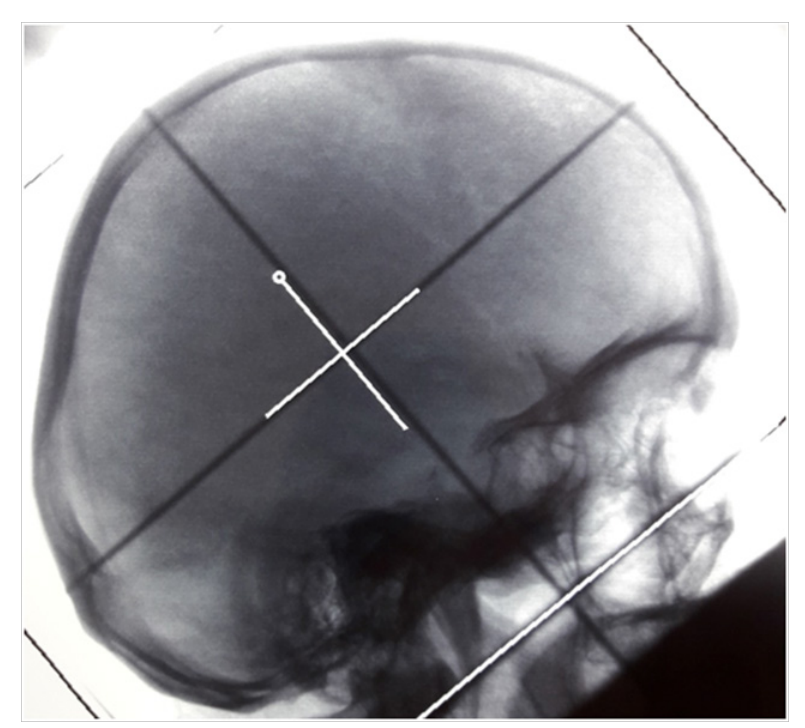

Figure 3 Whole brain - Orbital radiotherapy field.

\section{Discussion}

Metastases to the pituitary gland are considered unusual. ${ }^{2}$, representing $1 \%$ of the tumors in the pituitary gland, and 3-5\% of patients with carcinoma. ${ }^{10,16}$. Several tumors can metastasize in the pituitary gland, but the most common primary sites are lung and

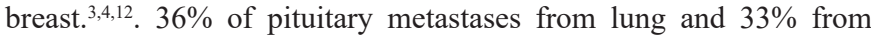
breast cancer. ${ }^{3,4,12}$.

The gastrointestinal tract, prostate, kidney, thyroid gland, pancreas, may be other sites of primary tumors which metastasize in the pituitary gland. ${ }^{1,5}$. The posterior lobe of the pituitary gland is the more often involved area, due to the richest blood supply.

The possible mechanisms for development of these metastases are the following:

a. Hematogenous spread

b. Direct extension from an adjacent affected bone

c. Meningeal spread through the suprasellar cistern. ${ }^{10,12,17}$

Metastases to the pituitary gland, are usually silent and only $7 \%$ are symptomatic. ${ }^{1,5}$ The most common manifestation is diabetes insipidus because of the posterior pituitary lobe involvement. Endocrine disorders due to anterior pituitary lobe tumor extension (e.g. hyperprolactinaemia,) retrobulbar headache, visual disturbances and ophthalmoplegia can also occur., ${ }^{3,7,12}$

The localization of the lesion in the pituitary stalk assists the differentiation between malignancy (e.g. metastasis) and benign causes (e.g. adenoma). The presence of diabetes insipidus, which may rarely be the first manifestation of metastatic disease, ${ }^{3,18}$ facilitates the differentiation between the two situations, ${ }^{3}$ as was the case we presented and which usually responds to desmopressin. Furthermore, significant increase of prolactin is found mainly in adenomas, while smaller increase appears when the stalk of the pituitary gland is involved. ${ }^{3,19}$ Upon MRI, pituitary metastases are isointense on T1 weighted images with high intensity on $\mathrm{T} 2$ weighted images, while adenomas tend to be isointense on $\mathrm{T} 1$ and $\mathrm{T} 2 .^{20,21}$ Suspicion of pituitary metastases is stronger when there is a progressive worsening of symptoms, increased age and history of malignancies. ${ }^{1,3}$ 
The management of pituitary metastases includes intensive local therapy, ${ }^{3,22}$ with surgical resection, conformal three dimensional radiotherapy and stereotactic radiotherapy combined with endocrine therapy and chemotherapy. ${ }^{23,24}$ In the case of visual disorders, surgical intervention can improve the symptoms. ${ }^{7,25}$

\section{Learning points}

a. Although pituitary metastases are rare, suspicion should be strong in patients with diabetes insipidus, headache, and visual disturbances.

b. Diabetes insipidus may rarely be the first manifestation of breast or lung cancer.

c. Prolactin levels and MRI findings can help in differentiation of pituitary adenoma from pituitary metastases.

d. Local control of the disease, with radiotherapy or surgery, is important, particularly to alleviate the symptoms.

\section{Acknowledgements}

None.

\section{Conflicts of Interest}

The authors declare that there is no conflict of interests regarding the publication of this paper.

\section{Patient consent}

The written informed consent of the patient was obtained, for the publication of her case.

\section{Funding}

None.

\section{References}

1. Young K, Beom L, Kyung L, et al. A Case of Pituitary, Metastasis from Breast Cancer that Presented as Left Visual Disturbance. J Korean Neurosurg Soc. 2012;51(2):94-97.

2. Kurkjian C, Armor JF, Kamble R, et al. Symptomatic metastases to the pituitary infundibulum resulting from primary breast cancer. Int J Clin Oncol. 2005;10(3):191-194.

3. Gormally JF, Izard MA, Robinson BG, et al. Pituitary metastasis from breast cancer presenting as diabetes insipidus. BMJ Case Rep. 2014;pii:bcr2014203683.

4. Asa SL. Practical pituitary pathology: what does the pathologist need to know? Arch Pathol Lab Med. 2008;132(8):1231-1240.

5. Teears RJ, Silverman EM (1975) Clinicopathologic review of 88 cases of carcinoma metastatic to the putuitary gland. Cancer. 1975;36(1):216220.

6. Hoellig A, Niehusmann P, Flacke S, et al. Metastasis to pituitary adenoma:case report and review of the literature. Cen Eur Neurosurg. 2009;70(3):149-153.
7. Sioutos P, Yen V, Arbit E. Pituitary gland metastases. Ann Surg Oncol. 1996;3(1):94-99.

8. Poursadegh Fard M, Borhani Haghighi A, Bagheri MH. Breast cancer metastasis to pituitary infandibulum. Iran J Med Sci. 2011;36(2):141144

9. Chaudhuri R, Twelves C, Cox TC, et al. MRI in diabetes insipidus due to metastatic breast carcinoma. Clin Radiol. 1992;46(3):184-188.

10. Spinelli GP, Lo Russo G, Miele E, et al. Breast cancer metastatic to the pituitary gland:a case report. World J Surg Oncol. 2013;10:137.

11. Suganuma H, Yoshimi T, Kita T, et al. Rare case with metastatic involvement of hypothalamo-pituitary and pineal body presenting as hypopituitarism and diabetes insipidus. Intern Med. 1994;33(12):795798.

12. Morita A, Meyer FB, Laws ER. Symptomatic pituitary metastases. $J$ Neurosurg. 1998;89(1):69-73.

13. Yi HJ, Kim $\mathrm{CH}$, Bak $\mathrm{KH}$, et al. Metastatic tumors in the sellar and parasellar regions: clinical review of four cases. J Korean Med Sci. 2000;15(3):363-367.

14. Dawood S, Broglio K, Gonzalez-Angulo AM, et al. Trends in survival over the past two decades among white and black patients with newly diagnosed stage IV breast cancer. J Clin Oncol. 2008;26(30):4891-4898.

15. Giordano SH, Buzdar AU, Smith TL, et al. Is breast cancer surviva improving? Cancer. 2004;100(1):44-52.

16. Fassett DR, Couldwell WT. Metastases to the pituitary gland. Neurosurg Focus. 2004;16(4):E8.6

17. Max MB, Deck MD, Rottenberg DA. Pituitary metastasis:incidence in cancer patients and clinical differentiation from pituitary adenoma. Neurology. 1981;31(8):998-1002.

18. Kimmel DW, O’Neill BP. Systemic cancer presenting as diabetes insipidus. Clinical and radiographic features of 11 patients with a review of metastatic-induced diabetes insipidus. Cancer. 1983;52(12):23552358 .

19. Levy A. Pituitary disease:presentation, diagnosis, and management. $J$ Neurol Neurosurg Psychiatry. 2004;75(3):47-52.

20. Komninos J, Vlassopoulou V, Protopapa D, et al. Tumors metastatic to the pituitary gland: case report and literature review. J Clin Endocrinol Metab. 2004;89(2):574-580.

21. Moses AM, Clayton B, Hochhauser L. Use of T1-weighted MR imaging to differentiate between primary polydipsia and central diabetes insipidus. AJNR Am J Neuroradiol. 1992;13(5):1273-1277.

22. Pagani O, Senkus E, Wood W, et al. International guidelines for management of metastatic breast cancer: can metastatic breast cancer be cured? J Natl Cancer Inst. 2010;102(7):456-463.

23. Lin CS, Lin SH, Chiang $\mathrm{YH}$, et al. Diabetes insipidus revealing an isolated pituitary stalk metastasis of breast cancer. European Journal of Neurology. 2007;14(7):11-12.

24. Murata Y, Ogawa Y, Yokoe I, et al. Pituitary stalk metastasis from breast cancer treated with systemic chemotherapy. Oncology Reports. 2003;10(6):1973-1975.

25. Chiang MF, Brock M, Patt S. Pituitary metastases. Neurochirurgia (Stuttg). 1990;33(4):127-131. 